HEDL-TME 75-141 UC-79b

CALCULATIONAL METHODS FOR

POWER CAPABILITY STUDIES

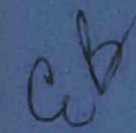

HANFORD ENGINEERING DEVELOPMENT LABORATORY

P.0. Box 1970 Richland, WA 99352

Operated by Westinghouse Hanford Company

Subsidiary of Westinghouse Electric Corporation

Prepared for the U.S. Energy Research and Development

- Administration under Contract No. E(45-1)-2170 


\section{DISCLAIMER}

This report was prepared as an account of work sponsored by an agency of the United States Government. Neither the United States Government nor any agency Thereof, nor any of their employees, makes any warranty, express or implied, or assumes any legal liability or responsibility for the accuracy, completeness, or usefulness of any information, apparatus, product, or process disclosed, or represents that its use would not infringe privately owned rights. Reference herein to any specific commercial product, process, or service by trade name, trademark, manufacturer, or otherwise does not necessarily constitute or imply its endorsement, recommendation, or favoring by the United States Government or any agency thereof. The views and opinions of authors expressed herein do not necessarily state or reflect those of the United States Government or any agency thereof. 


\section{DISCLAIMER}

Portions of this document may be illegible in electronic image products. Images are produced from the best available original document. 


\section{NOTICE}

This report was prepared as an account of work sponsored by the United States Government. Neither the United States nor the U.S. ERDA, nor any of their employees, nor any of their contractors, subcontractors, or their employees, makes any warranty, express or implied, or assumes any legal liability or responsibility for the accuracy, completeness or usefulness of any information, apparatus, product or process disclosed, or represents that its use would not infringe privately owned rights.

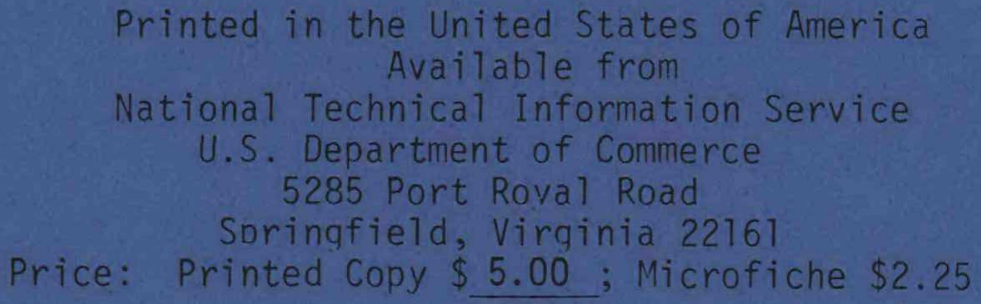


F. Schmittroth

February, 1976

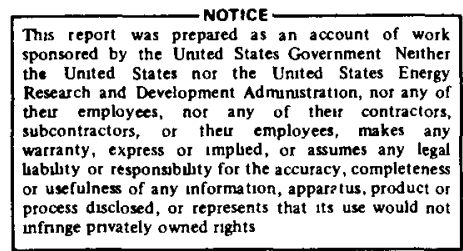

Hanford Engineering Development Laboratory

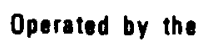

Westinghouse

Hanford Company
A Subsidiary of

Westinghouse Electric

Corporation for the United States Energy Rosoutreh and

Dovolopment Administration

Contract No. E(45-1)2170 
•

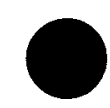


HEDL-TME 75-141

\title{
CALCULATIONAL METHODS FOR POWER CAPABILITY STUDIES
}

\author{
F. Schmittroth
}

\begin{abstract}
A formulation is developed to study reactor power capability based on the likelihood of incipient centerline fuel melting. Attention is also directed to the proper definition of this likelihood. As a consequence, it is shown how to compute the total probability for fuel melting as well as the expected number of fuel pellets and the associated variance. Factors considered include statistical variations in pellet fissile densitites, gap sizes, coolant temperatures, reactor power, and the power-to-melt function. General formulas suitable for computation are given along with more transparent analytical results.
\end{abstract}


-

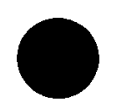




\section{CONTENTS}

Page

I. INTRODUCTION

II. FORMALISM 1

A. Introduction 1

B. Measures of Power Capability 3

C. Expected Number of Melted Pellets 4

D. Variance of the Number of Melted Pellets 6

E. Total Probability for Melting 8

III. CALCULATIONAL DETAILS 9

A. Expected Number of Melted Pellets 9

B. Variance of the Number of Melted Pellets 13

C. Total Probability for Melting 16

D. Analytical Considerations 17

$\begin{array}{ll}\text { IV. SUMMARY } & 24\end{array}$

$\begin{array}{ll}\text { REFERENCES } & 25\end{array}$

APPENDIX A A-1

FIGURES

Figure $\quad \underline{\text { Page }}$

1

1

A $A-1$ 


\section{CALCULATIONAL METHODS FOR POWER CAPABILITY STUDIES}

F. Schmittroth

\section{INTRODUCTION}

To meet the requirements for core performance and fuel management in the Fast Test Reactor (FTR) at Hanford, calculational tools are needed for power capability studies. One measure of power capability is the likelihood of incipient centerline melting of the fuel. (1) Based on statistical and probabalistic methods, the present work develops the formalism to calculate this likelihood as a function of various design and manufacturing variables, such as cladding temperatures and pellet fissile densities. Attention is directed toward two areas. First, in the restricted sense of fuel melting, it is desirable to reconfirm the power capability for the initial FTR cores and to maintain the tools needed to specify the loading for future cores. Secondly, with respect to economical core design and manufacture, as well as safe operation, it is important to identify the variables that affect fuel melting and to evaluate their relative importance.

The problem of defining a suitable measure of the likelihood of fuel melting is a key consideration. One measure, the expected number of melted pellets, may be small in two distinct ways. Even if the overall probability for some melting is near unity, the expected number of melted pellets can still be small if only a few pellets melt in each case. On the other hand, the melting can be rather extensive without a corresponding increase in the expected number of melted pellets so long as the total probability for melting in the first $\mathrm{place}$ is very small. To understand these relations, this work examines the total probability for melting and the variance of the number of melted pellets in addition to the expected number of melted pellets.

II. FORMALISM

A. Introduction

The basic power criterion assumed in this work is the onset of incipient melting of the fuel. Specifically, we desire some measure of the 
likelihood that the linear power, $L_{i}$, in some pellet, indexed by $i$, exceeds the power required for meiting, $M_{i}$ :

$$
L_{i} \geq M_{i}
$$

Since, clearly, the power to melt, $M_{j}$, is a function of various parameters, such as pellet gap size and clad temperature (see Appendix), we write

$$
M_{i}=M\left(g_{i}, t_{j}, x_{i}\right)
$$

where $g_{j}$ and $t_{j}$ denote the gap size and clad temperature (or other appropriate temperature measure), respectively, for the $i$-th pellet. The variable, $x_{i}$, denotes any other parameter that might affect the power to melt; gas pressures, for example.

Likewise, the linear power can be written as

$$
L_{i}=L+\Delta_{j}\left(d_{i}\right)
$$

where $L$ is the average linear power for the entire reactor and $\Delta_{i}\left(d_{i}\right)$ denotes the calculated spatial deviation from the average power as a function of the fissile density, $d_{j}$.

Because the detailed functional dependencies implied by Eqs. (2) and (3) are not known, we make the following expansions:

$$
\begin{aligned}
& L_{i}=L+\Delta_{i 0}+\frac{\partial \Delta_{i}}{\partial d_{i}}\left(d_{i}-d_{i 0}\right)+\cdots \\
& M_{i}=M_{0}+\frac{\partial M_{i}}{\partial g_{i}}\left(g_{i}-g_{0}\right)+\frac{\partial M_{i}}{\partial t_{i}}\left(t_{i}-t_{0}\right)+\frac{\partial M_{i}}{\partial x_{i}}\left(x_{i}-x_{0}\right)+\cdots,
\end{aligned}
$$

where $\Delta_{i 0}$ is the calculated spatial power correction at the nominal fissile density, $d_{i 0}$. Similarly, $M_{0}$ is the experimental ${ }^{(2)}$ power to melt at the 
nominal values, $g_{0}, t_{0}$, and $x_{0}$, for the gap size, clad temperature and any other pertinent variable, respectively.

There are uncertainties in all the above variables. Measurements of the reactor power, $L$, and the power to melt, $M_{0}$, are subject to errors. The manufacturing process can introduce uncertainties in the pellet parameters, $d_{i}$ and $g_{i}$. And there are calculational uncertainties in the power corrections, $\Delta_{i 0}$, and in the clad temperatures, $t_{j}$. Also, not only are the clad temperatures uncertain, the functional dependence of $M_{i}$ on them as given by $\frac{\partial M_{j}}{\partial t_{j}}$ is uncertain. This last source of uncertainty can be expressed as part of the uncertainty in the power to melt, $M_{0}$. The following list summarizes the notation for these variables, their mean values, and their associated one-sigma uncertainties:

$\begin{array}{llll}L & \bar{L} & \sigma_{L} & \text { measured linear power } \\ M_{0} & \bar{M}_{0} & \sigma_{M} & \text { experimental power to melt } \\ \Delta_{i 0} & \bar{\Delta}_{i 0} & \sigma_{\Delta} & \text { calculated power corrections } \\ g_{i} & \bar{g}_{i} & \sigma_{g} & \text { gap size } \\ d_{i} & \bar{d}_{i} & \sigma_{d} & \text { fissile density } \\ t_{i} & \bar{t}_{i} & \sigma_{t} & \text { clad temperature } \\ x_{i} & \bar{x}_{i} & \sigma_{x} & \text { other (gas, etc.). }\end{array}$

For some of these variables, the pellet gap, $g_{j}$, for instance, the expected value is the same as the value for which the power to melt, $M_{0}$, corresponds (i.e., $\bar{g}_{j}=g_{0}$ ). However, for other variables, such as the clad temperature, $t_{i}$, the expected value in the operating reactor will differ from the value in the power-to-melt experiment (i.e., $\bar{t}_{i}$ will differ from $\left.t_{0}\right)$.

\section{B. Measures of Power Capability}

A variety of measures are indicative of the melting that could occur if the reactor power is increased excessively. In this study, we review three: the expected number of melted pellets, the corresponding variance, and the total probability that some melting occurs. Before defining them more precisely, we turn to a simplified case to illustrate how these measures of the likelihood of melting differ. 
Suppose that a reactor has several zones with different power characteristics, a so-called hot zone and the rest, cold zones. Then if the reactor were fueled from a batch of pellets with randomly varying fissile densities, one could obtain an expected number of melted pellets, $\langle\bar{n}\rangle=1$, for example. Now take the same batch of fuel pellets as before but assume that all the pellets with higher fissile densities always reside in the same reactor zone. That is, the pellet fissile densities are correlated with their position. Because the chance that any given pellet will be placed in the hot zone is the same as before, the expected number of melted pellets will not change for the correlated case. Nevertheless, due to the correlation, when a pellet with high fissile density is placed in the hot zone, so will all the rest. Thus if melting does occur, it is more likely to be extensive. However, because all the pellets with a high fissile density are more likely to be placed in a colder zone, the total probability that some melting occurs will actually be decreased by the correlation. In addition, the variance of $n$ will be increased for the correlated case. For the uncorrelated case, one might expect about one melted pellet for every operation. But for the correlated case, most of the time no pellets would melt, while for part of the time, many pellets would melt.

C. Expected Number of Melted Pellets

As we shall see, the expected number of melted pellets is the most straightforward to calculate. First of all, we denote the probability that a particular pellet, $i$, melts for specified values of the linear power, $L_{i}$, and the power to melt, $M_{j}$, by $P\left(L_{i}-M_{j}\right)$, where $P(x)$ is the unit step function:

$$
P(x)= \begin{cases}1 & x \geq 0 \\ 0 & x<0 .\end{cases}
$$

If the linear power exceeds the power to melt, the pellet melts. Otherwise, it does not. Under these same conditions, the number of melted pellets is given by 


$$
n=\sum_{i} P\left(L_{i}-M_{i}\right)
$$

The effect of uncertainties in the independent variables which determine $L_{j}$ and $M_{j}$ can now be introduced. The uncorrelated effects are considered first. Uncertainties in variables, such as the average linear power, $L$, that affect all the pellets in a fully correlated manner are considered last. Therefore, to the extent that the fissile density and pellet gap variations are spatially uncorrelated, we can average over the appropriate distributions to obtain the probability, $\pi_{j}$, that any particular pellet melts:

$$
\pi_{i}=\int f_{d g}\left(d_{i}, g_{i}\right) P\left(L_{i}-M_{i}\right) d d_{i} d g_{i}
$$

where $f_{d g}$ describes the distribution of values for the fissile densities and pellet gaps. If there are no correlations between $d_{j}$ and $g_{j}$, one car write

$$
f_{d g}\left(d_{i}, g_{j}\right)=f_{d}\left(d_{i}\right) f_{g}\left(g_{i}\right)
$$

to simplify the description of these distributions. Because of the manufacturing process, these distributions are apt to be nonnormal. In practice, then, one could evaluate the integral in Eq. (8) using a Monte Carlo scheme to sample the appropriate distributions.

We can now average Eq. (7) over just the pellet variables to obtain

$$
\bar{n}=\sum_{i} \pi_{i}
$$

where the bar denotes the averaging process specified by Eq. (8). A11 the remaining variables $\left(L, M_{0}, \Delta_{i} \cdots\right)$ still have fixed values at this point.

Because there is some minimum subdivision of reactor zones that is useful to consider, we rewrite Eq. (10) as 


$$
\bar{n}=\sum_{\alpha} K_{\alpha} \pi
$$

where the variable, $\alpha$, now indexes the various zones, each of which contains $k_{\alpha}$ pellets. The expected value of $n$ averaged over the remaining variables is now given by

$$
\langle\bar{n}\rangle=\int f_{L} \int f_{M_{0}} \int f_{\Delta_{1}} \int f_{\Delta_{2}} \cdots \int f_{t_{1}} \int f_{t_{2}} \cdots\{\bar{n}\} d L d M_{0} \cdots
$$

By inspection, this complicated integral simplifies to

$$
\begin{aligned}
\langle\bar{n}\rangle & =\sum_{\alpha} k_{\alpha} \int f_{L} \int f_{M_{0}} \int f_{\Delta_{\alpha}} \int f_{t_{\alpha}}\left\{\pi_{\alpha}\right\} d L d M_{0} d \Delta_{\alpha 0} d t_{\alpha} \\
& =\sum_{\alpha} k_{\alpha}\left\langle\pi_{\alpha}^{\prime}\right\rangle .
\end{aligned}
$$

This important result shows that even though the variations in $L$ and $M_{0}$ affect the pellets in a fully correlated fashion, they can still be considered zone by zone, and similarly for the other variables. In other words, spatial correlations do not affect the expected number of melted pellets, $\langle\bar{n}\rangle$. Also, in contrast to the pellet variable distributions, $f_{d}$ and $f_{g}$, it is reasonable to assume that all the aistributions in Eq. (13) are normal. And because products of normal distributions are still normal, the four integrals and any others that one wants to consider collapse to a single integral. Examples of this technique are given in later sections.

D. Variance of the Number of Melted Pellets

The variance of the number of melted pellets is important if one wants a measure of the amount of melting in the event that melting occurs. If in nearly every operation a pellet melts, but rarely more than one, then the variance will be small. A large variance along with a small 
expected number is indicative of the situation where melting usually does not occur but may be extensive when it does happen. Mathematically, the variance is given by

$$
V(n)=\left\langle\overline{n^{2}}\right\rangle-\langle\bar{n}\rangle^{2}
$$

where $n$ is given by Eq. (7) and the bar and brackets have the same meanings as before. The bar denotes uncorrelated averages over the pellet variables [Eq. (8)] and the brackets denote global averages of the type given by Eq. (12).

To calculate $V(n)$, we first note the following relations:

$$
\begin{aligned}
\left\langle\overline{n^{2}}\right\rangle & =\left\langle\sum_{i j} P_{i} P_{j}\right\rangle \\
& =\left\langle\sum_{i j} \bar{P}_{i} \bar{P}_{j}+\sum_{i}\left(\overline{P_{i}^{2}}-\bar{P}_{i}^{2}\right)\right\rangle \\
& =\left\langle\left(\sum_{i} \pi_{i}\right)^{2}+\sum_{i}\left(\pi_{i}-\pi_{i}^{2}\right)\right.
\end{aligned}
$$

We then easily find

$$
\begin{aligned}
V(n)=\left\langle\left(\sum_{\alpha} K_{\alpha} \pi_{\alpha}\right)^{2}\right\rangle & +\sum_{\alpha} K_{\alpha}\left\langle\pi_{\alpha}-\pi_{\alpha}^{2}\right\rangle \\
& -\left(\sum_{\alpha} K_{\alpha}\left\langle\pi_{\alpha}\right\rangle\right)^{2}
\end{aligned}
$$

a result that can be rewritten as

$$
\left.V(n)=k^{2}\left[\langle\pi \dot{2}\rangle-\langle\pi\rangle^{2}\right]+k^{\prime}(\pi)\right\rangle
$$


where $\pi_{e}$ is an effective melting probability

$$
\pi_{e}=\frac{1}{K} \sum_{\alpha} K_{\alpha} \pi_{\alpha},
$$

with

$$
K=K_{\alpha} K_{\alpha} \cdot
$$

In obtaining Eq. (18) from Eq. (17) we neglected the term $\left\langle\pi_{\alpha}^{2}\right\rangle$ which should be much smaller than the term - $\pi_{\alpha} \backslash$. Unlike the expected number of melted pellets, the variance $V(n)$ is sensitive to spatial correlations. For this same reason, it is more difficult to compute, a topic we postpone temporariTy.

\section{E. Total Probability for Melting}

The total probability for melting is easily obtained from the individual melting probabilities. (3) If one is concerned only about whether or not melting is likely and not whether one, two, or more pellets melt, then the appropriate measure is the total probability for some melting to occur. This value is given by

$$
P_{T}=1-\left\langle{ }_{\alpha}^{\top}\left(1-\pi_{\alpha}\right) K_{\alpha} /\right.
$$

If $\pi_{\alpha}$ is small compared to one $\left(\pi_{\alpha}<<l\right)$ for all important values of the independent variables, one can use the approximation, $\left(1-\pi_{\alpha}\right)_{\alpha} \simeq \mathrm{e}^{-K_{\alpha} \pi_{\alpha}}$ to obtain

$$
\left\langle\mathrm{P}_{\mathrm{T}}\right\rangle \approx 1-\left\langle\mathrm{e}^{-\bar{n}}\right\rangle
$$

The probability, $P_{T}$, is also more difficult to calculate for the same reasons as for $V(n)$. 
III. CALCULATIONAL DETAILS

A. Expected Number of Melted Pellets

In order to assess the practicality of the methods just presented, we turn to specific details and assumptions in this section. We also consider what correlations can be accounted for and how they can be implemented. As noted above, the expected number of melted pellets is not influenced by spatial correlations and is the easiest to treat.

For convenience, Eq. (14), for the expected number of melted pellets, is rewritten here:

$$
\langle\bar{n}\rangle=\sum_{a} k_{\alpha}\left\langle\pi_{\alpha}\right\rangle
$$

where $\pi_{\alpha}$ is given by Eq. (8),

$$
\pi_{\alpha}=\int f_{d g}\left(d_{\alpha}, g_{\alpha}\right) P\left(L_{\alpha}-M_{\alpha}\right) d d_{\alpha} d g_{\alpha}
$$

Note that we have used the Greek subscript, $\alpha$, in place of the subscript, $i$, to indicate that a group of pellets with like characteristics are being considered. All pellets in a single group, $\alpha$, are assumed to be members of the same variable distributions.

Regardless of whether Eq. (8) is evaluated via Monte Carlo or whether Gaussian distributions are used, the functional dependence of $\pi_{\alpha}$ can be obtained from Eqs. (4) and (5) which can be combined as

$$
\begin{aligned}
L_{\alpha}-M_{\alpha} & =L+\Delta_{\alpha, 0}-M_{0}-\frac{\partial M_{\alpha}}{\partial t_{\alpha}}\left(t_{\alpha}-t_{0}\right) \\
& +\frac{\partial \Delta_{\alpha}}{\partial d_{\alpha}}\left(d_{\alpha}-d_{\alpha 0}\right)-\frac{\partial M_{\alpha}}{\partial g_{\alpha}}\left(g_{\alpha}-g_{0}\right)+\cdots
\end{aligned}
$$


After the integrations over $g_{\alpha}$ and $d_{\alpha}$ in Eq. (8) are done, $\pi_{\alpha}$ has the following form:

$$
\pi_{\alpha}=\pi\left(Z_{\alpha}\right)
$$

where

$$
Z_{\alpha}=L-M_{0}+\Delta_{\alpha 0}-D_{t}\left(t_{\alpha}-t_{0}\right)+D_{d}\left(\bar{d}_{\alpha}-d_{\alpha 0}\right)-D_{g}\left(\bar{g}_{\alpha}-g_{0}\right)+\cdots
$$

The coefficients, $D_{x}$, are constants given by the derivatives in Eq. (23), and $\bar{d}_{\alpha}$ and $\bar{g}_{\alpha}$ are the mean values of $d_{\alpha}$ and $g_{\alpha}$ as determined by the distribution, $f_{d g}$. As discussed previously, one calculates the spatial power corrections, $\Delta_{\alpha 0}$, for the expected fissile density, $\bar{d}_{\alpha}$, to give $\bar{d}_{\alpha}-d_{\alpha 0}=0$. Similarly, we have the condition, $\bar{g}_{\alpha}-g_{0}=0$, so that the last two terms in Eq. (25) are both zero. The explicit functional dependence of $\pi_{\alpha}$ becomes

$$
\pi_{\alpha}=\pi\left[L-M_{0}+\Delta_{\alpha 0}-D_{t}\left(t_{\alpha}-t_{0}\right)\right]
$$

where $\pi(Z)$ is a simple one-parameter function indicated schematically in Fig. 1 .

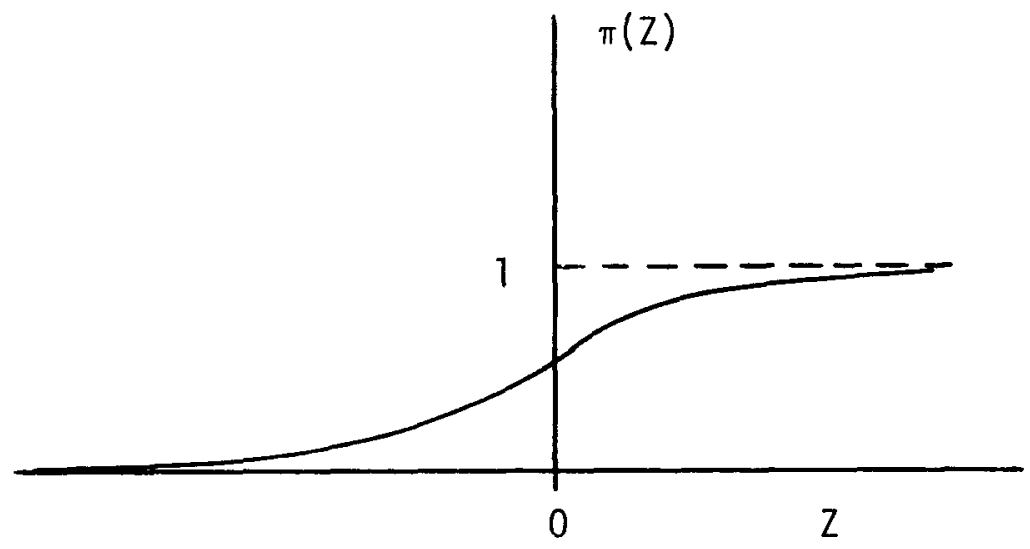

FIGURE 1. Single Pellet Melting Probability. 
Once the single function $\pi(Z)$ is computed, other corrections for clad temperature and spatial variations of the linear power are accounted for by a simple variable translation.

The remaining integrals implied by the brackets in $\left\langle\pi_{\alpha}\right\rangle$ and made explicit in Eq. (13) can now be easily done. Gaussian distributions are appropriate for the distributions in Eq. (13), and we write

$$
\begin{aligned}
f_{L} f_{M o} f_{\Delta_{\alpha 0}} f_{t_{\alpha}} & =G\left(L-\bar{L}, \sigma_{L}\right) G\left(M_{0}-\bar{M}_{0}, \sigma_{M}\right) \\
& \times G\left(\Delta_{\alpha 0}-\Delta_{\alpha 0}, \sigma_{\Delta}\right) G\left[D_{t}\left(t_{\alpha}-\bar{t}_{\alpha}\right), D_{t} \sigma_{t}\right],
\end{aligned}
$$

where

$$
G(x, \sigma)=\frac{1}{\sqrt{2 \pi} \sigma} e^{-\frac{x^{2}}{2 \sigma^{2}}} .
$$

From the fact that a product of Gaussians is in turn a Gaussian, we find

$$
F=f_{L} f_{M 0} f_{\Delta_{\alpha 0}} f_{t_{\alpha}}
$$

can be replaced by

$$
G\left(W-\bar{W}_{\alpha}, \sigma_{W}\right),
$$

where

$$
W_{\alpha}=L-M_{0}+\Delta_{\alpha 0}-D_{t} t_{\alpha},
$$

with

$$
\bar{W}_{\alpha}=\bar{L}-\bar{M}_{0}+\bar{\Delta}_{\alpha 0}-D_{t} \bar{t}_{\alpha} \text {, }
$$

and 


$$
\sigma_{W}^{2}=\sigma_{L}^{2}+\sigma_{M}^{2}+\sigma_{\Delta}^{2}+D_{t}^{2} \sigma_{t}^{2} \text {. }
$$

With these reductions, the expected value, $\left\langle\pi_{\alpha}\right\rangle$, is given by

$$
\left\langle\pi_{\alpha}\right\rangle=\int G\left(W_{\alpha}-\bar{W}_{\alpha}, \sigma_{W}\right) \pi\left[W_{\alpha}+D_{t} t_{0}\right] d W_{\alpha}
$$

which becomes, with a simple variable change,

$$
\left\langle\pi_{\alpha}\right\rangle=\int G\left(x, \sigma_{W}\right) \pi\left(x+S_{\alpha}\right) d x
$$

where

$$
S_{\alpha}=\bar{L}-\bar{M}_{0}+\bar{L}_{\alpha 0}+D_{t}\left(t_{0}-\bar{t}_{\alpha}\right)
$$

And finally, if one desires, the sum over $\alpha$ in Eq. (14) can be taken inside the integral:

$$
\langle\bar{n}\rangle=\int\left[G\left(x, \sigma_{W}\right) \Xi_{\alpha} K_{\alpha} \pi\left(x+S_{\alpha}\right)\right] d x
$$

so that only a single integration is needed.

In normal circumstances, the expected 1 inear power, $\left[+\Delta_{\alpha 0}\right.$, for zone, $\alpha$, should be well below the power to melt. Consequently, unless $\sigma_{W}$ is large, $x+S_{\alpha}$ will be negative and $\pi\left(x+S_{\alpha}\right)$ will be small. But if the expected clad temperature, $\bar{t}_{\alpha}$, is above the clad temperature, $t_{0}$, in the power-to-melt experiment, a positive term is added to $S_{\alpha}$ and $\pi$ will increase $\left(D_{t}\right.$ is negative since an increase in the clad temperature should decrease the power to melt). 
B. Variance of the Number of Melted Pellets

The required variance is given by Eqs. (18) and (19):

$$
V(n)=k^{2}\left[\pi_{e}^{2}-\left\langle\pi_{e}\right\rangle^{2}\right]+\left\langle k_{e}\right\rangle
$$

and

$$
K_{e}=\sum_{\alpha} K_{\alpha} \pi_{\alpha}
$$

Since the quantity, $\left\langle\mathrm{K}_{\mathrm{e}}\right.$, is the same as $\langle\bar{n}\rangle$, we need only to consider the term, $k^{2}\left\langle\pi e^{2}\right\rangle$.

The brackets, as defined by [see Eq. (12)]

$$
\left\langle\pi_{e}^{2}\right\rangle=\int f_{L} \int f_{M_{0}} \int f_{\Delta_{1}} \int f_{\Delta_{2}} \cdots \int f_{t_{1}} \int f_{t_{2}} \cdots\left\{\pi_{e}^{2}\right\} d L d M_{0} \cdots
$$

imply certain degrees of correlations. In the sense that all the pellets are affected in unison by changes in the global variables, $L$ and $M$, these variables give complete correlation. As Eq. (35) is written, variables such as $\Delta_{\alpha}$ and $t_{\alpha}$ give a lesser degree of correlation since they affect the pellets in different zones, $\alpha$, in an uncorrelated fashion. Although a substantial amount of simplification is possible, Eq. (35) still represents a considerable computational effort. Also, there is no special reason to assume that the variables, $\Delta_{\alpha}$ and $t_{\alpha}$, should be correlated over just the zones, $\alpha$. The primary reason for choosing a particular grouping of pellets into zones, is to adequately describe the spatial power variations, not to specify the degree of correlations. Consequently, we prefer to consider two limiting cases: first, where the effects of variations in the variables $\Delta_{\alpha}$ and $t_{\alpha}$ are completely uncorrelated, and secondly, where they are completely correlated. 
For the case of no correlations, the variables $\Delta_{\alpha}$ and $t_{\alpha}$ affect each of the pellets in an independent fashion, the same as we assumed earlier for the fissile density and gap distributions. The integrations over $\Delta_{\alpha}$ and $t_{\alpha}$ can be applied directly to $\pi_{\alpha}$ as given by Eq. (8) to give a quantity:

$$
\pi_{\alpha}^{\prime}=\int f_{\Delta_{\alpha 0}} \int f_{t_{\alpha}}\left\{\pi_{\alpha}\right\} d \Delta_{\alpha 0} d t_{\alpha},
$$

whose functional dependence is

$$
\pi_{\alpha}^{\prime}=\pi^{\prime}\left[L-M_{0}+\bar{\Delta}_{\alpha 0}-D_{t}\left(\bar{t}_{\alpha}-t_{0}\right)\right]
$$

In contrast to Eq. (26) for $\pi_{\alpha}$, the only statistical variables left in Eq. (37) are $L$ and $M_{0}$.

One can now form

$$
K \pi_{e}^{\prime}\left(W^{\prime}\right)=\sum_{\alpha} K_{\alpha} \pi^{\prime}\left[W^{\prime}+\bar{\Delta}_{\alpha 0}-D_{t}\left(\bar{t}_{\alpha}-t_{0}\right)\right]
$$

where

$$
W^{\prime}=L-M_{0}
$$

whose corresponding mean value and variance are

$$
\bar{W}^{\prime}=\bar{L}-\bar{M}_{0},
$$

and

$$
\sigma_{W}^{2}{ }^{\prime}=\sigma_{L}^{2}+\sigma_{M}^{2} .
$$


We now combine the Gaussian distributions for $L$ and $M_{0}$ as before to obtain

$$
\left\langle\pi_{e}^{2}\right\rangle=\int G\left(W^{\prime}-\bar{W}^{\prime}, \sigma_{W^{\prime}}\right) \pi_{e}^{\prime 2}\left(W^{\prime}\right) d W^{\prime} .
$$

The other limiting case to consider is where the variables, $\Delta_{\alpha 0}$ and $t_{\alpha}$, each behave in a fully correlated fashion. This condition can be expressed by the following definitions:

$$
\Delta_{\alpha 0}=\Delta+\bar{\Delta}_{\alpha 0},
$$

and

$$
t_{\alpha}=t+\bar{t}_{\alpha}
$$

where $\Delta$ and $\mathrm{t}$ are two new statistical variables whose mean values are zero and whose variances are $\sigma_{\Delta}^{2}$ and $\sigma_{t}^{2}$, respectively. One sees that all the values, $\Delta_{\alpha 0}$, vary in a coherent fashion as one varies $\Delta$, and similarly for t. In this instance, $\pi_{e}$ is given by

$$
K \pi_{e}\left(W^{\prime \prime}\right)=\sum_{\alpha} K_{\alpha} \pi\left[W^{\prime \prime}+\bar{\Delta}_{\alpha 0}-D_{t}\left(\bar{t}_{\alpha}-t_{0}\right)\right]
$$

where

$$
W^{\prime \prime}=L-M_{0}+\Delta-D_{t} t
$$

whose mean value is

$$
\bar{W}^{\prime \prime}=\bar{L}-\bar{M}_{0} \text {. }
$$

All four variables, $L, M_{0}, \Delta$, and $t$ can be combined into a single Gaussian distribution with variance, 


$$
\sigma_{W}^{2},=\sigma_{L}^{2}+\sigma_{W}^{2}+\sigma_{\Delta}^{2}+D_{t}^{2} \sigma_{t}^{2}
$$

so that one can again find $\left\langle\pi^{2}\right\rangle$ :

$$
\left.\left\langle\pi^{2}\right\rangle\right\rangle=\int G\left(W^{\prime \prime}-\bar{W}^{\prime \prime}, \sigma_{W^{\prime}}\right) \pi_{\mathrm{e}}^{2}\left(W^{\prime \prime}\right) d W^{\prime \prime} .
$$

Before concluding this section on variance, we briefly compare the correlated, Eq. (45), and uncorrelated, Eq. (40), expressions for $\left\langle\pi_{e}^{2}\right\rangle$. For the correlated case, the Gaussian width, $\sigma_{W^{\prime}}$, includes the variance, $\sigma_{\Delta}^{2}+D_{t}^{2} \sigma_{t}^{2}$, whereas for the uncorrelated case, this variance is included in the spreading of $\pi_{e}\left[\right.$ see Eq. (36)]. Notice that $\sigma_{W^{\prime}}$ and $\sigma_{W^{\prime}}$, are related by

$$
\sigma_{W^{\prime}{ }^{\prime}}^{2}=\sigma_{W^{\prime}}^{2}+\sigma_{\Delta}^{2}+D_{t}^{2} \sigma_{t}^{2}
$$

Although we have directed our attention in this section to correlations in variables, such as the linear power and clad temperatures, correlations in the pellet parameters can be treated in the same way. One would simply add another component to $\sigma_{W^{\prime}}$, in Eq. (45) to represent the correlated uncertainties [see Eq. (44C)].

\section{Total Probability for Melting}

The approximate expression, Eq. (22), for the total probability for melting can be reexpressed as

$$
{ }^{\prime} P_{T}, \approx 1-e^{-K \pi} e^{\prime}
$$

Exactly the same methods can be applied here ${ }_{-K}$ to evaluate $\left\langle\mathrm{P}_{\mathrm{T}}\right\rangle$ as was done for $\left\langle\pi^{2}\right\rangle$. The sole difference is that $e^{-K \pi}$ e replaces $\pi^{2}$ in Eqs. (40) and $(45)$. 


\section{Analytical Considerations}

In order to obtain further insight into the above formulas, we show how to proceed under certain simplifying assumptions. We calculate both the expected number of melted pellets as well as the corresponding variance where the clad gaps, $g_{\alpha}$, and fissile densities, $g_{\alpha}$, are uncorrelated but where the remaining variables are fully correlated.

To reduce the various integrals over Gaussian distributions, we first state the following preliminary result. Consider the integral

$$
I=\iint\left[G\left(x-x_{0}, \sigma_{x}\right) G\left(y-y_{0}, \sigma_{y}\right) f(a x+b y)\right] d x d y,
$$

where $G(x, \sigma)$ is the Gaussian function given by Eq. (28) and $f(w)$ is an arbitrary function. Then the following change of variable:

$$
\begin{gathered}
r=a x+b y \\
R=b\left(\frac{\sigma^{\prime}}{\sigma_{x}}\right) x-a\left(\frac{\sigma^{\sigma}}{\sigma_{y}}\right) y
\end{gathered}
$$

leads to

$$
I=\int G\left(r-r_{0}, \sigma_{r}\right) f(r) d r
$$

where

$$
\sigma_{r}^{2}=a^{2} \sigma_{x}^{2}+b^{2} \sigma_{y}^{2}
$$

and

$$
r_{0}=a x_{0}+b y_{0} .
$$


Equation (8) for $\pi_{\alpha}$ can now be written as

$$
\pi_{\alpha}=\iint\left[G\left(d_{\alpha}-d_{\alpha 0}, \sigma_{d}\right) G\left(g_{\alpha}-g_{0}, \sigma_{g}\right) P\left(L_{\alpha}-M_{\alpha}\right)\right] d d_{\alpha} d g_{\alpha}
$$

where

$$
L_{\alpha}-M_{\alpha}=Z_{\alpha}+D_{d}\left(d_{\alpha}-d_{\alpha 0}\right)-D_{g}\left(g_{\alpha}-g_{0}\right)
$$

with

$$
Z_{\alpha}=L-M_{0}+\Delta_{\alpha 0}-D_{t}\left(t_{\alpha}-t_{0}\right)
$$

From the definition of P, Eq. (6), and Eqs. (48)-(51), the result for $\pi_{\alpha}$ can be reduced to

$$
\pi_{\alpha}\left(z_{\alpha}\right)=\int_{-z_{\alpha}}^{\infty} \frac{1}{\sqrt{2 \pi} \sigma_{p}} e^{\frac{-r^{2}}{2 \sigma_{p}^{2}} d r}
$$

where

$$
\sigma_{\mathrm{p}}^{2}=D_{\mathrm{d}}^{2} \sigma_{\mathrm{d}}^{2}+D_{\mathrm{g}}^{2} \sigma_{\mathrm{g}}^{2}
$$

This result can be reexpressed as

$$
\pi_{\alpha}\left(z_{\alpha}\right)=\frac{1}{2} \operatorname{erfc}\left[-\frac{z_{\alpha}}{\sqrt{2} \sigma_{p}}\right]
$$

where erfc is the complementary error function with the following asymptotic expansion: 


$$
\pi_{\alpha}\left(Z_{\alpha}\right)=-\frac{\sigma_{p}}{\sqrt{2 \pi}} \frac{1}{Z_{\alpha}} e^{-\frac{Z_{\alpha}^{2}}{2 \sigma_{p}^{2}}}\left[1-\left(\frac{\sigma_{p}}{Z_{\alpha}}\right)^{2}+\cdots\right],
$$

valid for the condition, $-z_{\alpha}>>\sigma_{p}$.

If we keep only the first term in Eq. (60), Eq. (34) for $\langle\bar{n}\rangle$ can be immediately written as

$$
\langle\bar{n}\rangle \approx \int \frac{1}{\sqrt{2 \pi} \sigma_{w}} e^{-\frac{x 2}{2 \sigma_{w}^{2}}} K \pi_{e}(x) d x
$$

where

$$
\begin{gathered}
K_{e}(x) \approx \sum_{\alpha} k_{\alpha} \frac{\sigma_{p}}{\sqrt{2 \pi}}\left(\frac{-1}{x+S_{\alpha}}\right) e^{-\frac{\left(x+S_{\alpha}\right)^{2}}{2 \sigma_{p}^{2}},} \\
\sigma_{W}^{2}=\sigma_{L}^{2}+\sigma_{M}^{2}+\sigma_{\Delta}^{2}+D_{t}^{2} \sigma_{t}^{2},
\end{gathered}
$$

and

$$
S_{\alpha}=\bar{L}-\bar{M}_{0}+\bar{\Delta}_{\alpha 0}+D_{t}\left(t_{0}-\bar{t}_{\alpha}\right) .
$$

Equation (61) for $\langle\bar{n}\rangle$ is a sum of terms of the form

$$
T_{\alpha}=\int\left[\frac{1}{\sqrt{2 \pi} \sigma_{w}} e^{-\frac{x^{2}}{2 \sigma_{w}^{2}}} \frac{1}{\sqrt{2 \pi} \sigma_{p}} e^{-\frac{\left(x+S_{\alpha}\right)^{2}}{2 \sigma_{p}^{2}}} f(x)\right] d x
$$

where 


$$
f(x)=-\frac{K_{\alpha} \sigma_{p}^{2}}{x+S_{\alpha}}
$$

The integrand can be revised to give

$$
T_{\alpha}=\frac{1}{\sqrt{2 \pi} S} e^{-\frac{S^{2}}{2 S^{2}}} \int\left[\frac{1}{\sqrt{2 \pi} t} e^{-\frac{x^{2}}{2 t^{2}}} f\left(x-\alpha_{\alpha}\right)\right] d x
$$

where

$$
\begin{aligned}
& \frac{1}{t^{2}}=\frac{1}{\sigma_{p}^{2}}+\frac{1}{\sigma_{w}^{2}}, \\
& s^{2}=\sigma_{p}^{2}+\sigma_{w}^{2},
\end{aligned}
$$

and

$$
a_{\alpha}=\frac{S_{\alpha} \sigma_{W}^{2}}{S^{2}}
$$

So long as $t$ is small so that $f\left(x-a_{\alpha}\right)$ does not vary significantly in the integrand in Eq. (67), then we can approximate $f\left(x-a_{\alpha}\right)$ by $f\left(-a_{\alpha}\right)$ to give

$$
T_{\alpha} \approx \frac{1}{\sqrt{2 \pi} S} e^{-\frac{S_{\alpha}}{2 S^{2}}} f\left(-a_{\alpha}\right)
$$

Finally, we have

$$
\langle\bar{n}\rangle \approx \sum_{\alpha}\left(\frac{K_{\alpha} \sigma_{p}^{2}}{a_{\alpha}-S_{\alpha}}\right) \frac{1}{\sqrt{2 \pi} S} e^{-\frac{S_{\alpha}^{2}}{2 S^{2}}}
$$




$$
=\sum_{\alpha}\left(\frac{-K_{\alpha}}{\sqrt{2 \pi}}\right)\left(\frac{s}{s_{\alpha}}\right) e^{-\frac{s^{2}}{2 S^{2}}} .
$$

The same techniques can be used to evaluate the variance. For the case of full correlation in the variables, $\Delta_{\alpha}$ and $t_{\alpha}$, we need to compute

$$
\left\langle\pi^{2} \mathrm{e}\right\rangle=\int \mathrm{G}\left(W^{\prime}{ }^{\prime}-\bar{W}^{\prime}{ }^{\prime}, \sigma_{W^{\prime}}\right) \pi^{2} \mathrm{e}^{\left(W^{\prime}{ }^{\prime}\right) d W^{\prime} \cdot}
$$

where

$$
K_{e}=\sum_{\alpha} K_{\alpha} \pi\left[W^{\prime \prime}+\bar{\Delta}_{\alpha 0}-D_{t}\left(\bar{t}_{\alpha}-t_{0}\right)\right] .
$$

With the change of variable $x=W^{\prime \prime}-\bar{W}{ }^{\prime}$ ', this integral can be changed to

$$
\begin{gathered}
\left\langle\pi_{e}^{2}\right\rangle=\frac{2 \sigma_{p}^{\prime 3}}{\sqrt{2 \pi}} \sum_{\alpha \alpha^{\prime}} K_{\alpha} K_{\alpha^{\prime}} e^{-\frac{R_{\alpha \alpha^{\prime}}}{4 \sigma_{p}^{\prime 2}}} \\
x \int d x\left\{\frac{1}{\sqrt{2 \pi} \sigma_{w}} e^{-\frac{x^{2}}{2 \sigma_{w}^{2}}} \frac{1}{\sqrt{2 \pi} \sigma_{p}^{\prime}} e^{-\frac{\left(x+\overline{S_{\alpha \alpha^{\prime}}}\right)^{2}}{2 \sigma_{p}^{\prime 2}}}\left(\frac{1}{x+S_{\alpha}}\right)\left(\frac{1}{x+S_{\alpha}^{\prime}}\right)\right\}
\end{gathered}
$$

where

$$
\begin{aligned}
\sigma_{p}^{\prime} & =\frac{1}{\sqrt{2}} \sigma_{p}, \\
\overline{S_{\alpha \alpha^{\prime}}} & =\frac{1}{2}\left(S_{\alpha}+S_{\alpha^{\prime}}\right),
\end{aligned}
$$

and

$$
R_{\alpha \alpha}^{2}=\frac{1}{2}\left(S_{\alpha}-S_{\alpha^{\prime}}\right)^{2}
$$


This integral has a form identical to Eq. (65), and the same steps that led to Eq. (71) are appropriate. The result is

$$
\begin{array}{r}
\left\langle\pi_{e}^{2}\right\rangle \approx \frac{2 \sigma_{p}^{\prime 2}}{\sqrt{2 \pi}} \sum_{\alpha \alpha^{\prime}} K_{\alpha} K_{\alpha^{\prime}} e^{-\frac{R_{\alpha \alpha^{\prime}}}{4 \sigma_{p}^{\prime 2}}} \frac{1}{\sqrt{2 \pi} S^{\prime}} e^{-\frac{\overline{S_{\alpha \alpha^{\prime}}}}{2 S^{\prime 2}}} \\
\times\left(\frac{1}{S_{\alpha^{-}-a} a_{\alpha \alpha^{\prime}}}\right)\left(\frac{1}{S_{\alpha^{\prime}-a}-a_{\alpha \alpha^{\prime}}}\right)
\end{array}
$$

where

$$
S^{\prime 2}=\sigma_{p}^{\prime 2}+\sigma_{w}^{2}
$$

and

$$
a_{\alpha \alpha^{\prime}}=\frac{\overline{S_{\alpha \alpha^{\prime}}} \sigma_{W}^{2}}{S^{12}}
$$

The final factors can also be given as

$$
\left(S_{\alpha}-a_{\alpha \alpha^{\prime}}\right)\left(S_{\alpha^{\prime}}-a_{\alpha \alpha^{\prime}}\right)=\left(\frac{1}{2 S^{12}}\right)^{2}\left[\sigma_{p}^{4} S_{\alpha^{\prime}} S_{\alpha^{\prime}}-\sigma_{W}^{2}\left(\sigma_{W}^{2}+\sigma_{p}^{2}\right)\left(S_{\alpha}-S_{\alpha^{\prime}}\right)^{2}\right]
$$

The variance can now be easily obtained from Eq. (18).

These results are more transparent if we consider the case of a single zone. Then we have

$$
\left\langle\pi^{2}\right\rangle \approx \frac{2 \sigma_{p^{1}}^{2}}{\sqrt{2 \pi}} k_{j}{ }^{2} \frac{1}{\sqrt{2 \pi} S^{1}} e^{-\frac{S_{1}}{2 S^{12}}\left(\frac{2 S^{\prime 2}}{\sigma_{p}^{2} S_{1}}\right)^{2}}
$$




$$
=\frac{1}{2 \pi}\left(1+\frac{2 \sigma_{W}^{2}}{\sigma_{p}^{2}}\right)^{\frac{1}{2}}\left(1+\frac{\sigma_{W}^{2}}{S^{2}}\right)\left(\frac{S}{S_{1}}\right)^{2} e^{-\frac{S_{1}^{2}}{S^{2}\left(1+\sigma_{W}^{2} / S^{2}\right)}},
$$

which can be written as

$$
\left\langle\pi^{2}\right\rangle \approx \Gamma\langle\bar{n}\rangle^{2}
$$

where

$$
\Gamma=\left(1+\frac{2 \sigma_{w}^{2}}{\sigma_{p}^{2}}\right)^{\frac{1}{2}}\left(1+\frac{\sigma_{w}^{2}}{S^{2}}\right) e^{\frac{S_{T}^{2} \sigma_{w}^{2}}{S^{2}\left(S^{2}+\sigma_{w}^{2}\right)}} .
$$

From Eq. (18), the variance then becomes

$$
V(n)=(\Gamma-1)\langle\bar{n}\rangle^{2}+\langle\bar{n}\rangle .
$$

The simplest feature of this result is that the value of $\Gamma-1$ tends to zero as $\sigma_{W}^{2}$ goes to zero. Recall that $\sigma_{W}^{2}$ is the sum of variances, Eq. (63), for all the variables that affect the pellets coherently. If all these variances are small, then the variance of the number of melted pellets is approximately equal to the expected number of melted pellets, $\bar{n}\rangle$. Another point is that if the expected number of melted pellets is less than 1, the variance will be large only if the exponential in Eq. (84) is large, that is, if $e^{\gamma}$ is large where $\gamma$ is

$$
\gamma=\frac{S_{1}^{2} \sigma_{W}^{2}}{S^{2}\left(S^{2}+\sigma_{W}^{2}\right)}
$$

If there are no spatial power corrections or clad temperature corrections, then Eqs. (64) and (69) give 


$$
\gamma=\frac{\left(\bar{L}-\bar{M}_{0}\right)^{2} \sigma_{w}^{2}}{\left(\sigma_{p}^{2}+\sigma_{w}^{2}\right)\left(\sigma_{p}^{2}+2 \sigma_{w}^{2}\right)} .
$$

Untess $\sigma_{W}^{2}$ is near zero, $\gamma$ and hence $e^{\gamma}$, will be large if $\left[-\bar{M}_{0}\right.$ is appreciable. There are two distinct ways one might obtain a value of one for the expected number of melted pellets; either the case where one pellet was always close to the melting point, or where all of a large number of pellets were far from the melting point. So long as $\sigma_{w}$ is not very small, this latter case would have the larger variance.

A final limiting case is for $\sigma_{p}$ very smal1. The approximations that lead to Eq. (85) are not suitable in this case. However, as $\sigma_{p}$ goes to zero, $\pi(x)$ becomes a step function such that $\pi(x)=\pi^{2}(x)$. Then Eq. (17) gives

$$
\begin{aligned}
V(n) & \left.\left.=\cdot(K \pi)^{2}\right\rangle-(K, \pi\rangle\right)^{2} \\
& =(K-\bar{n}) \bar{n},
\end{aligned}
$$

a result that shows that the variance can become very large if $K$ is large, even if $\langle\vec{n}\rangle$ is not large.

IV. SUMMARY

In order to assess reactor power capability, a variety of formulas was derived to estimate the likelihood of incipient fuel melting. Specifically, we showed how to compute the expected number of melted pellets, $\bar{n}$, the variance of this quantity, $V(n)$, and the total probability for melting. Although numerical computations were not performed, we examined the role of a variety of factors including fuel pellet characteristics, such as clad gap and fissile density as well as reactor parameters, such as spatial power distributions and clad temperatures. All our results are 
based on the existence of a power-to-melt function, $M$, which gives the linear power at which incipient fuel melting occurs as a function of the various parameters.

The effect of various correlations was also considered. Although the formalism is general enough to treat a variety of correlations, our work was directed toward spatial correlations that affect all the pellets in unison, as in the case of the average linear power, $L$. In contrast to uncertainties in the clad gaps, for example, uncertainties in $L$ affect all the pellets in a uniform way. Correlations between various variables, for instance, clad gap and fissile density, require additional study. Nevertheless, it was felt that such correlations are relatively minor compared to other sources of uncertainty.

Equations suitable for both analytical and numerical studies were presented. However, although the analytical approximations for the expected number of melted pellets require a minimal amount of numerical effort, the analytical expressions for the variance are considerably more complicated. Nevertheless, several limiting cases were considered to provide insight into these results.

\section{References}

1. Alexander Sesonske, "Nuclear Power Plant Design Analys is," TID-26241, (NTIS, U.S. Department of Commerce; Springfield, Virginia, 1973), Chapter 4, pages 101-115.

2. R.D. Leggett et al, "Linear Heat Rating for Incipient Fuel Melting in $\mathrm{UO}_{2}-\mathrm{PuO}_{2}$ Fuel," Trans. Am. Nucl. Soc., 15 (1972), 752.

3. F. Schmittroth, Nucl. Sci. and Engr., 52 (1973), 95. 


\section{APPENDIX A}

\section{Power-to-Melt Function}

The power-to-melt function describes the linear power generation in a fuel pellet needed to raise the centerline temperature to the melting point. As expressed by

$$
M=M\left(g, t_{c}, x\right)
$$

the linear power, $M$, that causes melting is a function of several parameters; fuel-to-clad gap, $g$, coolant temperature, $t_{c}$, and others, $x$. In this section we review the functional dependence of $M$ on these parameters.

In cylindrical geometry, the heat flow per unit length, $g$, at a radius, $r$, is related to the radial temperature gradient by

$$
g=-(2 \pi r) k \frac{d T}{d r}
$$

where $k$ is the conductivity. This result is readily integrated to give the temperature drop through the fuel (see Fig. A):

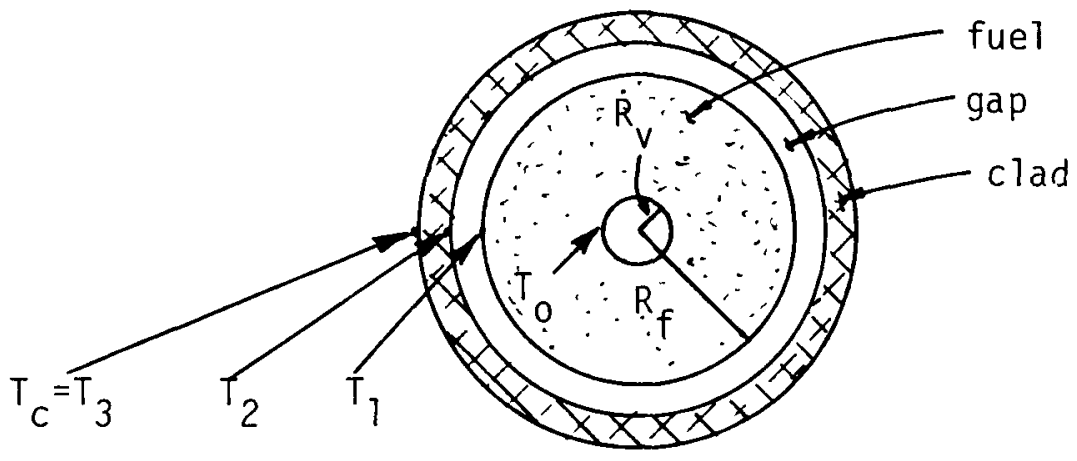

FIGURE A. Schematic Cross Section of an Oxide Fuel Pin. 


$$
T_{0}-T_{1}=\frac{L}{4 \pi k_{f}}\left[1+\frac{2 x^{2}}{1-x^{2}} \ln (x)\right]
$$

where $L$ is the linear power generation rate, $k_{f}$ is an average fuel conductivity, and $x$ is the ratio of the central void radius, $R_{v}$, to the fuel radius, $R_{f},\left(x=R_{v} / R_{f}\right)$. It is interesting to note that for typical small values of $x$, the second term in brackets in Eq. (A.3) is small compared to 1 , and the fuel temperature drop is independent of the fuel radius:

$$
T_{0}-T_{1} \approx \frac{L}{4 \pi k_{f}}
$$

In a similar fashion, one can obtain the temperature drop across the gap:

$$
T_{1}-T_{2} \approx \frac{g}{R_{f}}\left(\frac{L}{2 \pi k_{g}}\right),
$$

where $g$ is the gap size, and $k_{g}$ is an effective gap conductivity. $F i$ nally, the temperature drop across the cladding is given by

$$
T_{2}-T_{3} \approx \frac{L}{2 \pi k_{c}}\left(\frac{t}{R_{f}}\right)
$$

where $t$ is the clad thickness and $k_{c}$ is clad conductivity. The above results can now be added together to obtain the overall temperature drop between the centerline temperature, $T_{0}$, and the coolant temperature, $T_{c}=T_{3}:$

$$
\begin{aligned}
\Delta T & =T_{0}-T_{C} \\
& =\frac{L}{2 \pi}\left[\frac{1}{2 k_{f}}+\frac{g}{k_{g} R_{f}}+\frac{t}{k_{c} R_{f}}\right] .
\end{aligned}
$$


Although other phenomena can contribute to the temperature drop at the material interfaces, the following functional form for $\Delta T$ will still be valid so long as the additional terms are proportional to $L$ :

$$
\Delta T=(A+B g) L,
$$

where $A$ and $B$ are parameters (we note that B could depend on the gap, $g$, if the effective gap conductivity was a function of the gap size).

Since the power to melt is simply the value of the linear power, $L$, for which the centerline temperature, $T_{0}$, is equal to the melting point, $T_{M}$, of the oxide fuel, we can write

$$
M=\frac{T_{M}-T_{C}}{A+B g}
$$

for the power-to-melt function. For typical FTR fuel pins, $A$ is approximately 160 and $B$ is 10 where $g$ is given in mils, $T_{M}$ and $T_{C}$ in ${ }^{\circ} F$, and $M$ in $\mathrm{kw} / \mathrm{ft}$.

Equation (A.9) can be differentiated to determine the effect of variations in the underlying parameters on the power to melt. For instance, we can differentiate with respect to coolant temperature to obtain

$$
\frac{\Delta M}{M} \approx-\left(\frac{{ }^{T} C}{T_{M}-T_{C}}\right) \frac{\Delta T_{C}}{T_{C}} .
$$

For a coolant temperature of $1000^{\circ} \mathrm{F}$ and a melting temperature of $5000^{\circ} \mathrm{F}$, the factor $T_{C} /\left(T_{M}-T_{C}\right)$ is equal to $1 / 4$, a result independent of the values for $A$ and $B$. Therefore, a 10\% change in coolant temperature would alter the power-to-melt function by $10 / 4=2.5 \%$.

Variations in $M$ with respect to gap size can be obtained from 


$$
\frac{\partial M}{\partial g}=-\left(\frac{M}{A+B g}\right)\left(g \frac{d B}{d g}+B\right)
$$

Since $B$ is related to the gap conductivity by

$$
B=\frac{1}{2 \pi k_{g} R_{f}},
$$

then $d B / d g$ is zero unless the effective gap conductivity is not constant. In a similar fashion, the effect of varying gap conductivities (due to tag gas, for example) can also be determined. 
UC-79 (186)

$U C-79 b \quad(42)$

ERDA-RL (2)

A.G. Fremling

R.E. Constant

FFTF-PO (5)

R.L. Ferguson

RRD-HQ (2)

Director

HEDL (26)

R.A. Bennett

W.L. Bunch (2)

C.M. Cox

D.S. Dutt

E.A. Evans

R.D. Leggett

T.A. Mangelsdorf

J. Muroaka

J.V. Nelson

R.P. Omberg

R.E. Peterson

W.E. Roake

R.B. Rothrock

D.P. Schively

F. Schmittroth (2)

D.E. Simpson

A.E. Waltar

Central Records and Files (5)

Publications Services (2) 